\title{
ORBITS OF SEMIGROUPS OF TRUNCATED CONVOLUTION OPERATORS
}

\author{
STANISLAV SHKARIN \\ Queens's University Belfast, Pure Mathematics Research Centre, \\ University road, Belfast, BT7 1NN, UK \\ e-mail: s.shkarin@qub.ac.uk
}

(Received 9 September 2010; revised 9 June 2011; accepted 24 September 2011)

\begin{abstract}
We prove that a semigroup generated by finitely many truncated convolution operators on $L^{p}[0,1]$ with $1 \leqslant p<\infty$ is non-supercyclic. On the other hand, there is a truncated convolution operator, which possesses irregular vectors.
\end{abstract}

2010 Mathematics Subject Classification. 47A16, 37B99.

1. Introduction. Throughout the paper, all vector spaces are assumed to be over the field $\mathbb{K}$ being either the field $\mathbb{C}$ of complex numbers or the field $\mathbb{R}$ of real numbers, $\mathbb{Z}$ is the set of integers, $\mathbb{Z}_{+}$is the set of non-negative integers, $\mathbb{R}_{+}$is the set of non-negative real numbers and $\mathbb{N}$ is the set of positive integers. The symbol $L(X)$ stands for the space of continuous linear operators on a topological vector space $X$ and $X^{*}$ is the space of continuous linear functionals on $X$. A family $\mathcal{F}=\left\{F_{a}: a \in A\right\}$ of continuous maps from a topological space $X$ to a topological space $Y$ is called universal if there is $x \in X$ for which the orbit $O(\mathcal{F}, x)=\left\{F_{a} x: a \in A\right\}$ is dense in $Y$. Such an $x$ is called a universal element for $\mathcal{F}$. We use the $\operatorname{symbol} \mathcal{U}(\mathcal{F})$ to denote the set of universal elements for $\mathcal{F}$. If $X$ is a topological vector space, and $\mathcal{F}$ is a commutative subsemigroup of $L(X)$, then we call $\mathcal{F}$ hypercyclic if $\mathcal{F}$ is universal and the members of $\mathcal{U}(\mathcal{F})$ are called hypercyclic vectors for $\mathcal{F}$. We say that $\mathcal{F}$ is supercyclic if the semigroup $\mathcal{F}_{p}=\{z T: z \in \mathbb{K}, T \in \mathcal{F}\}$ is hypercyclic and the hypercyclic vectors for $\mathcal{F}_{p}$ are called supercyclic vectors for $\mathcal{F}$. An orbit $O\left(\mathcal{F}_{p}, x\right)$ will be called a projective orbit of $\mathcal{F}$. We refer to a tuple $\mathbf{T}=\left(T_{1}, \ldots, T_{n}\right)$ of commuting continuous linear operators on $X$ as hypercyclic (respectively, supercyclic) if the semigroup generated by $\mathbf{T}$ is hypercyclic (respectively, supercyclic). The concept of hypercyclic tuples of operators was introduced and studied by Feldman [5]. In the case $n=1$, it becomes the conventional hypercyclicity (or supercyclicity), which has been widely studied, see the book [1] and references therein.

Gallardo-Gutiérrez and Montes-Rodríguez [6], answering a question of Salas, proved that the Volterra operator

$$
V f(x)=\int_{0}^{x} f(t) d t,
$$

acting on $L_{p}[0,1]$ for $1 \leqslant p<\infty$, is non-supercyclic. This led to a quest of finding supercyclic or even hypercyclic operators as close as possible to the Volterra operator. In [9], it is observed that $L^{2}[0,1]$ admits a norm $\|\cdot\|$ defining a weaker topology such that $V$ is $\|\cdot\|$-continuous and the continuous extension of $V$ to the completion of 
$\left(L^{2}[0,1],\|\cdot\|\right)$ is hypercyclic. The mainstream of the quest dealt with searching of hypercyclic or supercyclic operators commuting with $V$.

Truncated convolution operators form an important class of operators commuting with $V$. Let $C_{0}[0,1]$ be the Banach space of continuous functions $f:[0,1] \rightarrow \mathbb{C}$ satisfying $f(0)=0$ and carrying the sup-norm and let $\mathbf{M}$ be the space of finite $\sigma$ additive $\mathbb{K}$-valued Borel measures $\mu$ on $[0,1)$. For $\mu \in \mathbf{M}$, we consider the operator $C_{\mu} \in L\left(C_{0}[0,1]\right)$ acting according to the formula

$$
C_{\mu} f(x)=\int_{0}^{1} f_{x}(t) d \mu, \text { where } f_{x}(t)=f(x-t) \text { if } t \leqslant x \text { and } f_{x}(t)=0 \text { if } t>x .
$$

In other words, $C_{\mu} f$ is the restriction to $[0,1]$ of the convolution of $f$ and $\mu$. According to the well-known properties of convolutions, $\left\|C_{\mu} f\right\|_{p} \leqslant\|\mu\|\|f\|_{p}$ for every $f \in C_{0}[0,1]$, where $\|\mu\|$ is the total variation of $\mu$ and $\|f\|_{p}$ is the norm of $f$ in $L^{p}[0,1]$ for $1 \leqslant p \leqslant \infty$. Thus, $C_{\mu}$ extends uniquely to a continuous linear operator on $L^{p}[0,1]$ for $1 \leqslant p<\infty$ and the norm of this operator does not exceed $\|\mu\|$. The same holds for $L^{\infty}[0,1]$ : the obstacle of $C_{0}[0,1]$ being non-dense in $L^{\infty}[0,1]$ can easily be overcome by either using the density of $C_{0}[0,1]$ in $L^{\infty}[0,1]$ in $*$-weak topology and $*$-weak continuity of $C_{\mu}$ or by simply restricting to the non-closed invariant subspace $L^{\infty}[0,1]$ of the extension of $C_{\mu}$ to $L^{1}[0,1]$. This allows to treat each $C_{\mu}$ as a member of each $L\left(L^{p}[0,1]\right)$. From the basic properties of convolutions, it also follows that the set

$$
\mathbb{A}=\left\{C_{\mu}: \mu \in \mathbf{M}\right\}
$$

of truncated convolution operators is a commutative subalgebra of $L\left(C_{0}[0,1]\right)$ and of each $L\left(L^{p}[0,1]\right)$. For instance, $C_{\mu} C_{v}=C_{\eta}$, where $\eta$ is the restriction to $[0,1)$ of the convolution of $\mu$ and $\nu$. Since $V=C_{\lambda}$ with $\lambda$ being the Lebesgue measure on $[0,1)$, A consists of operators commuting with $V$. It is worth noting [10] that on $L^{1}[0,1]$, $C_{0}[0,1]$ and $L^{\infty}[0,1]$, there are no other continuous linear operators commuting with $V$, while this fails for $L^{p}[0,1]$ with $1<p<\infty$.

In [7,9], it is shown that $V$ is not weakly supercyclic (=non-supercyclic on $L^{p}[0,1]$ carrying the weak topology). In $[3, \mathbf{4}, \mathbf{7}]$, it is demonstrated that certain truncated convolution operators are not weakly supercyclic. Léon-Saavedra and PiquerasLerena [7] raised a question whether any $T \in L\left(L^{p}[0,1]\right)$ commuting with $V$ is not weakly supercyclic. This question was answered affirmatively in [14]. Still, there remained a possibility of existence of a hypercyclic or at least supercyclic tuple of truncated convolution operators.

THEOREM 1.1. Let $T_{1}, \ldots, T_{n} \in$ A. Then, for any $f \in L^{1}[0,1]$, the projective orbit

$$
\left\{w T_{1}^{k_{1}} \ldots T_{n}^{k_{n}} f: k_{j} \in \mathbb{Z}_{+}, w \in \mathbb{K}\right\}
$$

is nowhere dense in $L^{1}[0,1]$ equipped with the weak topology.

The usual comparing the topologies argument provides the following corollary:

COROLLARY 1.2. There are no tuples of truncated convolution operators weakly supercyclic when acting on $L^{p}[0,1]$ with $1 \leqslant p<\infty$.

Since only truncated convolution operators commute with $V$ acting on $L^{1}[0,1]$, the following result holds: 
COROLLARY 1.3. There are no weakly supercyclic tuples of operators on $L^{1}[0,1]$ commuting with $V$.

Our method applies not only to finitely generated semigroups. For example, it also takes care of the semigroup of the Riemann-Liouville operators, which form a sub-semigroup of $\mathbb{A}$. Namely,

$$
V^{z} f(x)=\frac{1}{\Gamma(z)} \int_{0}^{x} f(t)(x-t)^{z-1} d t \text { with } z \in \Pi=\{z \in \mathbb{C}: \operatorname{Re} z>0\},
$$

where $\Gamma$ is the Euler's gamma function. Of course, to consider $V^{z}$ with non-real $z$, we need the underlying space to be over $\mathbb{C}$. Clearly, $V^{z}=C_{\mu_{z}}$ with $\mu_{z}$ being the absolutely continuous measure on $[0,1)$ with the density $a_{z}(x)=\frac{x^{z-1}}{\Gamma(z)}$. Since $a_{z} \in L^{1}[0,1]$ for every $z \in \Pi$, each $V^{z}$ is a truncated convolution operator and therefore belongs to $\mathbb{A}$. Moreover, it is easy to verify that $V^{z} V^{w}=V^{z+w}$ for every $z, w \in \Pi$ and $V=V^{1}$. Thus, $\left\{V^{z}\right\}_{z \in \Pi}$ is a semigroup and $V^{n}$ is exactly the $n$th power of $V$, which justifies the notation $V^{z}$. The map $z \mapsto V^{z}$ from $\Pi$ to $L\left(L^{p}[0,1]\right)$ is operator norm continuous and holomorphic. Thus, $\left\{V^{z}\right\}_{z \in \Pi}$ is a holomorphic operator norm continuous semigroup of operators acting on $L^{p}[0,1]$. In [13], it is shown that for every $\alpha \in(0, \pi / 2)$, the subsemigroup $\left\{V^{r e^{i \theta}}: r>0,-\alpha<\theta<\alpha\right\}$ is non-supercyclic on $L^{p}[0,1]$ for $1 \leqslant p<\infty$. We prove the following stronger result:

Proposition 1.4. For every $f \in L^{1}[0,1]$, the set $\left\{w V^{z} f: z \in \Pi, w \in \mathbb{C}\right\}$ is nowhere dense in $L^{1}[0,1]$ with respect to the weak topology. In particular, the semigroup $\left\{V^{z}\right\}_{z \in \Pi}$ is not weakly supercyclic.

In order to compensate for the lack of chaotic behaviour of the orbits of operators commuting with $V$ in terms of the density in the underlying space, we show that these operators can exhibit chaotic behaviour in terms of the norms of the members of the orbit. The following definition is due to Beauzamy [2]. Let $X$ be a Banach space and $x \in X$. We say that $x$ is an irregular vector for $T \in L(X)$ if $\underline{\lim }_{n \rightarrow \infty}\left\|T^{n} x\right\|=0$ and $\overline{\lim }_{n \rightarrow \infty}\left\|T^{n} x\right\|=\infty$. The concept of irregularity was studied by Prajitura [11]. It is worth noting that Smith [15] constructed a non-hypercyclic continuous linear operator $T$ on a separable Hilbert space such that each non-zero vector is irregular for $T$.

THEOREM 1.5. There are a truncated convolution operator $T$ and $f \in C_{0}[0,1]$ such that

$$
\varliminf_{n \rightarrow \infty}\left\|T^{n} f\right\|_{\infty}=0 \text { and } \varlimsup_{n \rightarrow \infty}\left\|T^{n} f\right\|_{1}=\infty .
$$

In particular, $f$ is an irregular vector for $T$ acting on $L^{p}[0,1]$ for each $p \in[1, \infty]$.

2. Obstacles to weak supercyclicity. In this section, we develop techniques for the proof of Theorem 1.1 and prove Proposition 1.4. We say that a topological vector space $X$ carries a weak topology if the topology of $X$ is the weakest topology making each $f \in Z$ continuous, where $Z$ is a fixed linear space of linear functionals on $X$ separating the points of $X$. Of course, any weak topology is locally convex. Moreover, $X^{*}=Z$ if $X$ carries the weak topology defined by the space $Z$ of functionals. As usual, when speaking of the weak topology on a given topological vector space, we always mean the 
weak topology defined by $X^{*}$ with $X^{*}$ being the dual of $X$ with respect to the original topology of $X$.

We say that a subset $A$ of a topological space $X$ is somewhere dense if it is not nowhere dense.

The following lemma exhibits a feature of weak topologies. Its conclusion fails, for example, for infinite-dimensional Banach spaces. Recall that a subset $B$ of a vector space $X$ is called balanced if $\lambda x \in B$ for every $x \in B$ and $\lambda \in \mathbb{K}$ such that $|\lambda| \leqslant 1$.

LEMMA 2.1. Let $X$ and $Y$ be topological vector spaces with weak topology and $A: X \rightarrow Y$ be a continuous linear operator with dense range. Then, $A(M)$ is somewhere dense in $Y$ for every $M$ somewhere dense in $X$.

Proof. Since $A$ is continuous, it is enough to show that $A(U)$ is somewhere dense in $Y$ for every non-empty open subset $U$ of $X$. Since $A$ is linear and translation maps on a topological vector space are homeomorphisms, it suffices to verify that $A(U)$ is somewhere dense in $Y$ for every neighbourhood $U$ of 0 in $X$. It is easy to see that the sets of the shape

$$
U=\left\{x \in X:\left|A^{*} g_{j}(x)\right|<1,\left|f_{k}(x)\right|<1 \text { for } 1 \leqslant j \leqslant m, 1 \leqslant k \leqslant n\right\}
$$

form a basis of neighbourhoods of 0 in $X$, where $g_{1}, \ldots, g_{m}$ are linearly independent functionals in $Y^{*}$ and $f_{1}, \ldots, f_{n} \in X^{*}$ are such that $f_{k}+A^{*}\left(Y^{*}\right)$ are linearly independent in $X^{*} / A^{*}\left(Y^{*}\right)$. Note that $A^{*}$ is injective since $A$ has dense range, and therefore the functionals $A^{*} g_{j}$ are also linearly independent. Thus, it suffices to show that $A(U)$ is somewhere dense in $Y$ for $U$ defined in the above display. Clearly,

$$
\begin{array}{r}
A(U)=W \cap V, \text { where } W=\left\{y \in Y:\left|g_{j}(y)\right|<1 \text { for } 1 \leqslant j \leqslant m\right\} \\
\text { and } V=\left\{A x:\left|f_{k}(x)\right|<1 \text { for } 1 \leqslant k \leqslant n\right\} .
\end{array}
$$

Since $W$ is a non-empty open subset of $Y$, the job will be done if we verify that $V$ is dense in $Y$. Assume the contrary. Since $V$ is convex and balanced, the Hahn-Banach theorem implies that there is a non-zero $f \in Y^{*}$ such that $|f(y)|<1$ for each $y \in V$. That is, $|f(A x)|=\left|A^{*} f(x)\right|<1$ whenever $\left|f_{k}(x)\right|<1$ for $1 \leqslant k \leqslant n$. It follows that $A^{*} f$ is a linear combination of $f_{k}$. Since $A^{*}$ is injective, $A^{*} f \neq 0$, and therefore a non-trivial linear combination of $f_{k}$ belongs to $A^{*}\left(Y^{*}\right)$. We have arrived to a contradiction, which completes the proof.

LEMMA 2.2. Let $K$ be a compact subset of an infinite-dimensional topological vector space $X$ such that $0 \notin K$. Then, $\Lambda=\{\lambda x: \lambda \in \mathbb{K}, x \in K\}$ is a closed nowhere dense subset of $X$.

Proof. Closeness of $\Lambda$ in $X$ is a straightforward exercise. Assume that $\Lambda$ is somewhere dense. Since $\Lambda$ is closed, its interior $L$ is non-empty. Since $K$ is closed and $0 \notin K$, we can find a non-empty balanced open set $U$ such that $U \cap K=\varnothing$. Clearly, $\lambda x \in L$ whenever $x \in L$ and $\lambda \in \mathbb{K}, \lambda \neq 0$. Since $U$ is open and balanced, the latter property of $L$ implies that the open set $W=L \cap U$ is non-empty. Taking into account the definition of $\Lambda$, the inclusion $L \subseteq \Lambda$, the equality $U \cap K=\varnothing$ and the fact that $U$ is balanced, we see that every $x \in W$ can be written as $x=\lambda y$, where $y \in K$ and $\lambda \in \mathbb{D}=\{z \in \mathbb{K}:|z| \leqslant 1\}$. Since both $K$ and $\mathbb{D}$ are compact, $Q=\{\lambda y: \lambda \in \mathbb{D}, y \in K\}$ is a compact subset of $X$. Since $W \subseteq Q, W$ is a non-empty open set with compact closure. Since such a set exists [12, p. 23] only if $X$ is finite dimensional, the proof is complete. 
Now we can prove Proposition 1.4. Its proof resembles the proof of the main result in [14] and gives an idea of the proof of Theorem 1.1 in the following sections. For $f \in L^{1}[0,1]$, we say that the infimum of the support of $f$ is 0 if for every $\varepsilon>0, f$ does not vanish (almost everywhere) on $[0, \varepsilon]$.

LEMMA 2.3. Let $f, g \in L^{1}[0,1]$ be such that the infima of the supports off and $g$ are 0 . Then, there exist truncated convolution operators $C, B \in L\left(L^{1}[0,1]\right)$ injective and with dense range such that $C f=B g$.

Proof. Let $\mu$ and $\nu$ be the absolutely continuous measures on $[0,1]$ with the densities $g$ and $f$, respectively. Applying the Titchmarsh theorem on the supports of convolutions to $\mu * v$, we see that $C_{\mu}, C_{\nu}$ and their duals are injective. Thus, $C_{\mu}$ and $C_{\nu}$ are both injective and have dense ranges. Next, $C_{\mu} f$ and $C_{\nu} g$ both equal to the restriction to $[0,1]$ of the convolution $f * g$. Thus, $C_{\mu} f=C_{\nu} g$ and therefore $C=C_{\mu}$ and $B=C_{v}$ satisfy all required conditions.

Proof of Proposition 1.4. Let $f \in L^{1}[0,1]$. If $f$ vanishes (almost everywhere) on $[0, \varepsilon]$ for some $\varepsilon \in(0,1)$, then each $V^{z} f$ belongs to the space $L$ of $g \in L^{1}[0,1]$ vanishing on $[0, \varepsilon]$. Since $L$, being a proper closed linear subspace of $L^{1}[0,1]$, is nowhere dense (in the weak topology), the result follows. It remains to consider the case when the infimum of the support of $f$ is 0 . Consider the multiplication operator $M \in L\left(L^{1}[0,1]\right)$, $M h(x)=x h(x)$. It is straightforward to verify that

$$
V^{z} M-M V^{z}=-z V^{z+1} \text { for every } z \in \Pi .
$$

Clearly, the infimum of the support of $M f$ is also 0. By Lemma 2.3, there exist truncated convolution operators $B, C \in L\left(L^{1}[0,1]\right)$ injective and with dense range such that $C M f=B f$. Assume that Proposition 1.4 does not hold. That is, the set $\Omega=\left\{w V^{z} f: z \in \Pi, w \in \mathbb{C}\right\}$ is somewhere dense in $L^{1}[0,1]$ carrying the weak topology. By Lemma 2.1, $V(\Omega)=\left\{w V^{z+1} f: z \in \Pi, w \in \mathbb{C}\right\}$ is also somewhere dense in $L^{1}[0,1]$ with weak topology. Applying (2.1) with $z$ replaced by $z+1$ to $f$ and multiplying by $C$ from the left, we get $C V^{z+1} M f-C M V^{z+1} f=-(z+1) C V^{z+2} f$. Using the commutativity of $\mathbb{A}$, we obtain $V^{z+1} C M f-C M V^{z+1} f=-(z+1) C V V^{z+1} f$. Since $C M f=B f$, we have

$$
V^{z+1} B f-C M V^{z+1} f=-(z+1) C V V^{z+1} f .
$$

Using commutativity of $\mathbb{A}$ once again, we arrive to

$$
(C M-B) V^{z+1} f=(z+1)(C V) V^{z+1} f \text { whenever } \operatorname{Re} z>-1 .
$$

Pick any non-zero $g \in L^{1}[0,1]$, which lies in the interior of the closure of $\left\{w V^{z+1} f\right.$ : $z \in \Pi, w \in \mathbb{C}\}$ in the weak topology. Since $C V$ is injective, $C V g \neq 0$ and we can pick $\varphi \in\left(L^{1}[0,1]\right)^{*}=L^{\infty}[0,1]$ such that $\varphi(C V g)=(C V)^{*} \varphi(g) \neq 0$. Take $c>0$ such that $\left|(C M-B)^{*} \varphi(g)\right|<c\left|(C V)^{*} \varphi(g)\right|$ and consider the weakly open set

$$
W=\left\{h \in L^{1}[0,1]:\left|(C M-B)^{*} \varphi(h)\right|<c\left|(C V)^{*} \varphi(h)\right|\right\} .
$$

By Lemma 2.2, the set $\left\{w V^{z+1} f: \operatorname{Re} z \geqslant 0,|z| \leqslant c, w \in \mathbb{C}\right\}$ is nowhere dense in $L^{1}[0,1]$ with the weak topology. Since $g \in W$ and $g$ lies in the interior of the closure of $\left\{w V^{z+1} f: z \in \Pi, w \in \mathbb{C}\right\}$ in the weak topology, we can find $w \in \mathbb{C} \backslash\{0\}$ and $z \in \Pi$ 
such that $|z|>c$ and $w V^{z+1} f \in W$. Using (2.2), we have

$$
(C M-B)^{*} \varphi\left(w V^{z+1} f\right)=(z+1)(C V)^{*} \varphi\left(w V^{z+1} f\right) .
$$

Since $w V^{z+1} f \in W$, we have

$$
\left|(C M-B)^{*} \varphi\left(w V^{z+1} f\right)\right|<c\left|(C V)^{*} \varphi\left(w V^{z+1} f\right)\right| .
$$

By the last two displays, $|z| \leqslant|z+1|<c$ and we have arrived to a contradiction.

The proof of Theorem 1.1 goes along the same lines as the proof of Proposition 1.4. However, we need some extra preparation. A strongly continuous operator semigroup $\left\{T^{[t]}\right\}_{t \in G}$ on a topological vector space $X$ is a collection of continuous linear operators $T^{[t]}$ on $X$ labelled by the elements of an additive sub-semigroup $G$ of $\mathbb{R}^{n}$ containing 0 and such that $T^{[0]}=I, T^{[t+s]}=T^{[t]} T^{[s]}$ for any $t, s \in G$ and the map $t \mapsto T^{[t]} x$ from $G$ to $X$ is continuous for each $x \in X$, where $G$ carries the topology inherited from $\mathbb{R}^{n}$. If $n=k+m$ and $G=\mathbb{R}_{+}^{k} \times \mathbb{Z}_{+}^{m}$, then for the sake of brevity, we shall call a strongly continuous operator semigroup $\left\{T^{[t]}\right\}_{t \in G}$, an operator $(k, m)$-semigroup on $X$. In this case, we will often write $T_{j}$ with $1 \leqslant j \leqslant n$ instead of $T^{\left[e_{j}\right]}$, where $e_{j}$ is the $j$ th basic vector in $\mathbb{R}^{n}$ and we shall write $T_{j}^{s}$ instead of $T^{\left[s e_{j}\right]}$. In this notation, $T^{[t]}=T_{1}^{t_{1}} \ldots T_{n}^{t_{n}}$.

LEMmA 2.4. Let $X$ be an infinite-dimensional topological vector space, $x \in X, c>0$ and $\left\{T^{[t]}\right\}_{t \in \mathbb{R}_{+}^{k} \times \mathbb{Z}_{+}^{m}}$ be an operator $(k, m)$-semigroup on $X$. Then, the set

$$
\Omega_{c}=\left\{w T^{[t]} x: w \in \mathbb{K}, t_{j} \leqslant c \text { for } 1 \leqslant j \leqslant n\right\}
$$

is nowhere dense in $X$.

Proof. First, observe that the general case is easily reduced to the case $m=0$. Indeed, it follows from the fact that the union of finitely many nowhere dense sets is nowhere dense. Thus, we can assume that $m=0$. If $x$ is not a cyclic vector for $\left\{T^{[t]}\right\}_{t \in \mathbb{R}_{+}^{k}}$, $\Omega_{c}$ is contained in a proper closed linear subspace of $X$, and therefore is nowhere dense. Thus, we can assume that $x$ is cyclic for $\left\{T^{[t]}\right\}_{t \in \mathbb{R}_{+}^{k}}$. Without loss of generality, we can also assume that there is $l \in\{1, \ldots, k\}$ such that $T_{j}(X)$ is dense in $X$ if $j \geqslant l$ and $T_{j}(X)$ is not dense in $X$ if $j<l$.

Claim 1. For every $s \in \mathbb{R}_{+}^{k}, T^{[s]} x=0$ if and only if $T^{[s]}=0$.

Proof. Assume the contrary. Then, there is $s \in \mathbb{R}_{+}^{k}$ such that $T^{[s]} \neq 0$ and $T^{[s]} x=0$. Then, $x \in L=\operatorname{ker} T^{[s]} \neq X$. Since the linear space $L$ is invariant for every $T^{[t]}$ and contains $x$, the linear span of the orbit of $x$ with respect to $\left\{T^{[t]}\right\}_{t \in \mathbb{R}_{+}^{k}}$ is contained $L$. Since the latter is a proper closed linear subspace of $X$, we have arrived to a contradiction with the cyclicity of $x$ for $\left\{T^{[t]}\right\}_{t \in \mathbb{R}_{+}^{k}}$.

Claim 2. For every $s \in \mathbb{R}_{+}^{k}, T^{[s]}=0$ if and only if $T_{1}^{s_{1}} \ldots T_{l-1}^{S_{l-1}}=0$ (if $l=1$, we have the empty product, which is always assumed to be $I$ ).

Proof. Since $T_{j}(X)$ is dense in $X$ for $j \geqslant l, B(X)$ is dense in $X$, where $B=T_{l}^{s_{l}} \ldots T_{k}^{s_{k}}$. Since $T^{[s]}=A B$ with $A=T_{1}^{s_{1}} \ldots T_{l-1}^{s_{l-1}}$ and $A B=B A$, the density of the range of $B$ implies that $T^{[s]}=0$ if and only if $A=0$.

Since $x \neq 0$ and $\left\{T^{[t]}\right\}_{t \in \mathbb{R}_{+}^{k}}$ is strongly continuous, we can pick $\varepsilon \in(0, c)$ such that $T_{1}^{\varepsilon} \ldots T_{l-1}^{\varepsilon} x \neq 0$. By Claims 1 and $2, T^{[t]} x \neq 0$ whenever $t_{j} \leqslant \varepsilon$ for $j<l$. Thus, the 
compact set

$$
K=\left\{T^{[t]} x: t_{j} \leqslant \varepsilon \text { if } j<l \text { and } t_{j} \leqslant c \text { if } j \geqslant l\right\}
$$

does not contain 0. By Lemma 2.2,

$$
\Omega=\left\{w T^{[t]} x: w \in \mathbb{K}, t_{j} \leqslant \varepsilon \text { if } j<l \text { and } t_{j} \leqslant c \text { if } j \geqslant l\right\}
$$

is closed and nowhere dense in $X$. On the other hand,

$$
\Omega_{c} \backslash \Omega \subseteq \bigcup_{j<l} T_{j}^{\varepsilon}(X),
$$

and therefore $\Omega_{c} \backslash \Omega$ is nowhere dense in $X$ since $\overline{T_{j}^{\varepsilon}(X)} \neq X$ for $j<l$. Hence, $\Omega_{c}$ is nowhere dense as the union of the nowhere dense sets $\Omega$ and $\Omega_{c} \backslash \Omega$.

REMARK. In the above proof, we have repeatedly used the elementary fact that if $\left\{T^{t}\right\}_{t \geqslant 0}$ is a strongly continuous operator semigroup, then $T^{t}$ for $t>0$ either all have dense ranges or all have non-dense ranges.

LEMMA 2.5. Let $X$ be an infinite-dimensional topological vector space carrying a weak topology, $\mathbb{B}$ be a commutative subalgebra of $L(X), x \in X,\left\{T^{[t]}\right\}_{t \in \mathbb{R}_{+}^{k} \times \mathbb{Z}_{+}^{m}}$ be an operator $(k, m)$-semigroup on $X$ such that each $T^{[t]}$ has dense range and belongs to $\mathbb{B}$, $M \in L(X), B, C \in \mathbb{B},\left[T_{j}, M\right]=S_{j} \in \mathbb{B}$ for $1 \leqslant j \leqslant n=k+m, C M x=B x, C(X)$ is dense in $X$ and the convex span of the operators $R_{1}, \ldots, R_{n}$ does not contain the zero operator, where

$$
R_{j}=T_{1} \ldots T_{j-1} S_{j} T_{j+1} \ldots T_{n} .
$$

Then, $O=\left\{w T^{[t]} x: w \in \mathbb{K}, t \in \mathbb{R}_{+}^{k} \times \mathbb{Z}_{+}^{m}\right\}$ is nowhere dense in $X$.

Proof. Observe that

$$
[A B, M]=B[A, M]+A[B, M] \text { if } A, B,[A, M],[B, M] \in \mathbb{B} .
$$

It follows that $\left[T_{j}^{r}, M\right]=r T_{j}^{r-1} S_{j}$ whenever $r \in \mathbb{N}$ and $j>k$. Similarly, it is easy to check that $\left[T_{j}^{r}, M\right]=r T_{j}^{r-1} S_{j}$ whenever $r \geqslant 1$ is rational and $j \leqslant k$. By strong continuity, $\left[T_{j}^{r}, M\right]=r T_{j}^{r-1} S_{j}$ whenever $r \geqslant 1$ is real and $j \leqslant k$. Applying the above display once again, we arrive to

$$
\left[T^{[t+1]}, M\right]=\left(\left(t_{1}+1\right) R_{1}+\cdots+\left(t_{n}+1\right) R_{n}\right) T^{[t]} \text { for every } t \in \mathbb{R}_{+}^{k} \times \mathbb{Z}_{+}^{m},
$$

where $R_{j}$ are defined in (2.3) and $\mathbf{1}=(1, \ldots, 1)$. For $t \in \mathbb{R}_{+}^{k} \times \mathbb{Z}_{+}^{m}$, let $N(t)=n+t_{1}+$ $\cdots+t_{n}$ and $\lambda(t)=\left(\frac{t_{1}+1}{N(t)}, \ldots, \frac{t_{n}+1}{N(t)}\right) \in \mathbb{R}^{n}$ and for $\lambda \in \mathbb{R}^{n}$, let $R_{[\lambda]}=\lambda_{1} R_{1}+\ldots+\lambda_{n} R_{n}$. Then, for every $t \in \mathbb{R}_{+}^{k} \times \mathbb{Z}_{+}^{m}, R_{[\lambda(t)]}$ is a convex combination of $R_{j}$. In this notation, the above display can be rewritten as

$$
\left[T^{[t+1]}, M\right]=N(t) R_{[\lambda(t)]} T^{[t]} .
$$

Multiplying the equality in the above display by $C$ from the left and applying the result to $x$, we obtain $C T^{[t+1]} M x-C M T^{[t+1]} x=N(t) C R_{[\lambda(t)]} T^{[t]} x$. Since $C$ commutes with 
each $T^{[s]}$, we get

$$
T^{[t+1]} C M x-C M T^{[t+1]} x=N(t) C R_{[\lambda(t)]} T^{[t]} x .
$$

Since $C M x=B x$ and $B$ commutes with each $T^{[s]}$, we arrive to

$$
D T^{[t]} x=N(t) C R_{[\lambda(t)]} T^{[t]} x \text { for each } t \in \mathbb{R}_{+}^{k} \times \mathbb{Z}_{+}^{m} \text {, where } D=(B-C M) T^{[1]} .
$$

Assume that Lemma 2.5 does not hold. That is, the interior $W$ of the closure of $O$ in $X$ is non-empty. From the definitions of $O$ and $W$, it follows that there is $s \in \mathbb{R}_{+}^{k} \times \mathbb{Z}_{+}^{m}$ such that $T^{[s]} x \in W$. Next, we observe that the convex span $K$ of the vectors $C T^{[s]} R_{1} x, \ldots, C T^{[s]} R_{1} x$ does not contain 0 . Indeed, assume that it is not the case. Then, there are $\lambda_{1}, \ldots, \lambda_{n} \in \mathbb{R}_{+}$such that $\lambda_{1}+\cdots+\lambda_{n}=1$ and $C T^{[s]} R_{[\lambda]} x=0$. Since 0 is not in the convex span of $R_{j}, R_{[\lambda]} \neq 0$. Since $C$ and $T^{[s]}$ have dense ranges and commute with $R_{[\lambda]}, A=C T^{[s]} R_{[\lambda]} \neq 0$. Since $A$ commutes with each $T^{[t]}, \operatorname{ker} A$ is invariant for each $T^{[t]}$. Since $x \in \operatorname{ker} A$, we have $O \subseteq \operatorname{ker} A$, and therefore $O$ is nowhere dense in $X$ because ker $A$ is a proper closed subspace of $X$. Thus, 0 does not belong to the convex compact set $K$. By the Hahn-Banach theorem [12, p. 46], there is $f \in X^{*}$ such that $\operatorname{Re} f(y)>1$ for every $y \in K$. In particular,

$$
\operatorname{Re} f\left(C T^{[s]} R_{j} x\right)=\operatorname{Re} C^{*} R_{j}^{*} f\left(T^{[s]} x\right)>1 \text { for } 1 \leqslant j \leqslant n .
$$

Let $c=\left|D^{*} f\left(T^{[s]} x\right)\right|+1$. Then, the open set

$$
U=\left\{v \in X:\left|D^{*} f(v)\right|<c, \operatorname{Re} R_{j}^{*} C^{*} f(v)>1 \text { for } 1 \leqslant j \leqslant n\right\}
$$

contains $T^{[s]} x$ and therefore $U \cap W$ is non-empty. By Lemma 2.4, the set $O_{c}=\left\{w T^{[t]} x\right.$ : $w \in \mathbb{K}, N(t) \leqslant c\}$ is nowhere dense in $X$. Since $O$ is dense in $U \cap W$ and $O_{c}$ is nowhere dense, $O \backslash O_{c}$ intersects $U \cap W$. Thus, we can pick $z \in \mathbb{K}$ and $t \in \mathbb{R}_{+}^{k} \times \mathbb{Z}_{+}^{m}$ such that $N(t)>c$ and $u=z T^{[t]} x \in U \cap W$. Applying $f$ to the both sides of (2.4), we obtain $D^{*} f(u)=N(t) R_{[\lambda(t)]}^{*} C^{*} f(u)$. Hence,

$$
N(t) \operatorname{Re} R_{[\lambda(t)]}^{*} C^{*} f(u)=\operatorname{Re} D^{*} f(u) \leqslant\left|D^{*} f(u)\right| .
$$

Since the real number $\operatorname{Re} R_{[\lambda(t)]}^{*} C^{*} f(u)$ is in the convex span of the numbers $\operatorname{Re} R_{j}^{*} C^{*} f(u)$, each of which is in $(1, \infty)$ (because $u \in U)$, we have $\operatorname{Re} R_{[\lambda(t)]}^{*} C^{*} f(u)>1$. The inclusion $u \in U$ also implies that $\left|D^{*} f(u)\right|<c$. Thus, by the above display, $N(t)<c$, which is a contradiction.

In order to apply Lemma 2.5 to prove Theorem 1.1, we need more information on truncated convolution operators.

3. Elementary properties of truncated convolution operators. Throughout this section, when speaking of $C_{\mu}$, we assume that it acts on $C_{0}[0,1]$ or on $L^{p}[0,1]$ with $1 \leqslant p<\infty$.

First, observe that $C_{\mu}=I$ precisely when $\mu=\delta$, where $\delta$ is the point mass at $0: \delta(\{0\})=1$ and $\delta(A)=0$ if $0 \notin A$. As we have already mentioned, the Titchmarsh theorem on supports of convolutions implies that $C_{\mu}$ and $C_{\mu}^{*}$ are injective if $\inf \operatorname{supp}(\mu)=0$. Hence, $C_{\mu}$ has dense range if $\inf \operatorname{supp}(\mu)=0$. In the case inf supp $\mu=a>0$, the same theorem ensures that $C_{\mu}$ is nilpotent with the order of nilpotency being the first natural number $n$ for which $n a \geqslant 1$. If $\mu(\{0\})=0$, then $\mu$ 
is the variation norm limit of its restrictions $\mu_{n}$ to $\left[2^{-n}, 1\right]$. Hence, $C_{\mu}$ is the operator norm limit of the sequence $C_{\mu_{n}}$ of nilpotent operators. Thus, $C_{\mu}$ is quasi-nilpotent if $\mu(\{0\})=0$. It immediately follows that the spectrum $\sigma\left(C_{\mu}\right)$ is the singleton $\{\mu(\{0\})\}$ for each $\mu \in \mathbf{M}$. Recall that a power $T^{n}$ of an operator $T$ is the identity $I$ if and only if $T$ is the direct sum of operators of the shape $c I$ with $c^{n}=1$. In the case when the spectrum of $T$ is a singleton, this means that $T=c I$ with $c^{n}=1$. The above observations are summarized in the following proposition:

Proposition 3.1. Let $\mu \in \mathbf{M}$. Then,

(3.1.1) $C_{\mu}$ is injective if and only if $C_{\mu}$ has dense range if and only if inf $\operatorname{supp}(\mu)=0$;

(3.1.2) $C_{\mu}^{n}=a I$ if and only if $C_{\mu}=c I$ (equivalently, $\mu=c \delta$ ) with $c^{n}=a$;

(3.1.3) $\sigma\left(C_{\mu}\right)=\{\mu(\{0\})\}$.

We need some extra information on truncated convolution operators.

Lemma 3.2. $T \in \mathbb{A}$ is invertible if and only if $T=c e^{A}$ with $c \in \mathbb{K} \backslash\{0\}$ and $A \in \mathbb{A}$ being quasinilpotent.

Proof. Of course, $c e^{A}$ belongs to $\mathbb{A}$ and is invertible if $c \in \mathbb{K} \backslash\{0\}$ and $A \in \mathbb{A}$. Assume now that $T \in \mathbb{A}$ is invertible. By (3.1.3), $T=C_{\mu}$ with $c=\mu(\{0\}) \neq 0$. Thus, $\mu=c \delta+v$, where $v \in \mathbf{M}$ and $v(\{0\})=0$. That is, $T=c(I+S)$, where $S=\frac{1}{c} C_{v} \in \mathbb{A}$ is quasi-nilpotent. Since $S$ is quasi-nilpotent, the operator

$$
A=\ln (I+S)=\sum_{n=1}^{\infty} \frac{(-1)^{n-1}}{n} S^{n}
$$

is well-defined, belongs to $\mathbb{A}$ and is also quasi-nilpotent. It remains to observe that $T=c e^{A}$.

LEMMA 3.3. Let $\left\{T^{t}\right\}_{t \geqslant 0}$ be a strongly continuous operator semigroup such that each $T^{t}$ belongs to $\mathbb{A}$ and $T^{1}$ is invertible. Then, there are a quasi-nilpotent $A \in \mathbb{A}$ and $a \in \mathbb{K} \backslash\{0\}$ such that $T^{t}=e^{t a} e^{t A}$ for $t \in \mathbb{R}_{+}$.

Proof. By Lemma 3.2, there are $c \in \mathbb{K} \backslash\{0\}$ and $A \in \mathbb{A}$ quasi-nilpotent such that $T^{1}=c e^{A}$. Then, for every $k, m \in \mathbb{N},\left(T^{k / m} e^{-k A / m}\right)^{m}=c^{k} I$. By (3.1.2), $T^{k / m} e^{-k A / m}$ is a scalar multiple of the identity. Thus, $T^{t} e^{-t A}$ is a scalar multiple of the identity whenever $t \in \mathbb{R}_{+}$is rational. By strong continuity, $T^{t} e^{-t A}$ is a scalar multiple of the identity for each $t \in \mathbb{R}_{+}$. Thus, there is a function $\alpha: \mathbb{R}_{+} \rightarrow \mathbb{K} \backslash\{0\}$ such that $T^{t} e^{-t A}=\alpha(t) I$ for $t \in \mathbb{R}_{+}$. Since $\left\{T_{t}\right\}$ and $\left\{e^{-t A}\right\}$ are strongly continuous operator semigroups, whose members commute, $\{\alpha(t) I\}$ is a strongly continuous operator semigroup as well. Hence, $\alpha$ is continuous, $\alpha(0)=1$ and $\alpha(t+s)=\alpha(t) \alpha(s)$ for every $t, s \in \mathbb{R}_{+}$. It follows that there is $a \in \mathbb{K}$ such that $\alpha(t)=e^{t a}$ for $t \in \mathbb{R}_{+}$. Thus, $T^{t}=e^{t a} e^{t A}$ for each $t \in \mathbb{R}_{+}$.

Let now $M$ be the operator of multiplication by the argument acting on the same space as the truncated convolution operators:

$$
M h(x)=x h(x)
$$

Lemma 3.4. Let $\mu \in \mathbf{M}$. Then, the commutator $\left[C_{\mu}, M\right]$ belongs to $\mathbb{A}$. Moreover, $\left[C_{\mu}, M\right]=C_{\mu^{\prime}}$, where $\mu^{\prime} \in \mathbf{M}$ is the measure absolutely continuous with respect to $\mu$ with the density $\rho(x)=-x$. 
Proof. It is easy to verify that the set of $\mu \in \mathbf{M}$ satisfying $\left[C_{\mu}, M\right]=C_{\mu^{\prime}}$ is closed in $\mathbf{M}$ with respect to the weak topology $\sigma$ provided by the natural dual pairing (M, $C[0,1])$. Thus, it is enough to prove the required equality for $\mu$ from a $\sigma$-dense set in M. As such a set, we can take the set of absolutely continuous measures with polynomial densities. By linearity, it suffices to prove the equality $\left[C_{\mu}, M\right]=C_{\mu^{\prime}}$ for $\mu$ being absolutely continuous with the density $d(x)=x^{n}$ for $n \in \mathbb{Z}_{+}$. In the latter case, the required equality is an elementary integration by parts exercise (left to the reader).

Since $\mu^{\prime}=0$ if and only if $\mu=c \delta$ with $c \in \mathbb{K}$ and $C_{\delta}=I$, we arrive to the following corollary:

COROLlary 3.5. The equality $\left[C_{\mu}, M\right]=0$ holds if and only if $C_{\mu}=c I$ with $c \in \mathbb{K}$.

The operator $M$ is needed in order to apply Lemma 2.5 to prove Theorem 1.1. The trickiest part of such an application is due to the fact that the condition of 0 being not in the convex span of the operators $R_{j}$ may fail for operator semigroups contained in A. The next lemma allows us to determine exactly when does this condition fail.

3.1. Main lemma. Recall that for a non-zero finite Borel $\sigma$-additive complex valued measure $\mu$ on $\mathbb{R}$ with compact support, its Fourier transform

$$
\widehat{\mu}(z)=\int_{\mathbb{R}} e^{-i t z} d \mu(t)
$$

is an entire function of exponential type [8, p. 84] bounded on the real axis. Moreover, the numbers $a=\inf \operatorname{supp}(\mu)$ and $b=\sup \operatorname{supp}(\mu)$ determine the indicator function of $\widehat{\mu}$. Namely,

$$
h_{\mu}(\theta)=\varlimsup_{r \rightarrow+\infty} \frac{\ln \left|\widehat{\mu}\left(r e^{i \theta}\right)\right|}{r}= \begin{cases}b \sin \theta, & \text { if } \theta \in[0, \pi], \\ a \sin \theta, & \text { if } \theta \in(-\pi, 0) .\end{cases}
$$

Furthermore, by the Cartwright theorem [8, p. 243], $\widehat{\mu}$ is of completely regular growth on each ray $\left\{r e^{i \theta}: r>0\right\}$ with $\theta \in(-\pi, 0) \cup(0, \pi)$. That is, for every $\theta \in(-\pi, 0) \cup$ $(0, \pi)$, there is an open set $E_{\theta} \subset(0, \infty)$ such that

$$
\lim _{\substack{r \rightarrow+\infty \\ r \in E_{\theta}}} \frac{\ln \left|\widehat{\mu}\left(r e^{i \theta}\right)\right|}{r}=h_{\mu}(\theta) \text { and } \lim _{r \rightarrow+\infty} \frac{\lambda\left([0, r] \cap E_{\theta}\right)}{r}=1 \text {, }
$$

where $\lambda$ is the Lebesgue measure on $\mathbb{R}$. Recall that a subset of $\mathbb{R}_{+}$satisfying the second equality in (3.2) is said to have density 1 . Thus, the completely regular growth condition means that upper limit in the definition of the indicator function turns into the limit if we restrict ourselves to $r$ from a suitable open set of density 1 .

LEMMA 3.6. Let $\mu_{1}, \ldots, \mu_{n}$ be finite Borel $\sigma$-additive complex valued measure on $\mathbb{R}$ with compact support satisfying inf $\operatorname{supp}\left(\mu_{j}\right)=0$ for $1 \leqslant j \leqslant n$. For each $j \in\{1, \ldots, n\}$, let

$$
v_{j}=\mu_{1} * \ldots * \mu_{j-1} * \mu_{j}^{\prime} * \mu_{j+1} * \ldots * \mu_{n},
$$


where $*$ is the convolution and $\mu^{\prime}$ denotes the measure absolutely continuous with respect to $\mu$ with the density $\rho(x)=-x$. Also assume that $c_{1}, \ldots, c_{n}>0$ and

$$
\inf \operatorname{supp}(v)>0 \text {, where } v=c_{1} v_{1}+\ldots+c_{n} v_{n} .
$$

Then, $\mu_{j}(\{0\}) \neq 0$ for $1 \leqslant j \leqslant n$.

Proof. Assume the contrary. Then, without loss of generality, we can assume that $\mu_{1}(\{0\})=0$. We can also assume that $c_{j}>1$ for every $j$. Indeed, multiplying all $c_{j}$ by the same positive number does not change anything.

Since $\mu_{1}(\{0\})=0, \mu_{1}$ is the variation norm limit of the sequence $\left\{\mu_{1, n}\right\}_{n \in \mathbb{N}}$ of the restrictions of $\mu_{1}$ to $\left[2^{-n}, 1\right]$. Let $\alpha \in(0, \pi / 2)$ and $A_{\alpha}=\left\{r e^{i \theta}: r \geqslant 0, \theta \in[\alpha-\pi,-\alpha]\right\}$. By definition of the Fourier transform, each $\widehat{\mu_{1, n}}(z)$ converges to 0 as $|z| \rightarrow \infty$ for $z$ from the angle $A_{\alpha}$. Moreover, $\widehat{\mu_{1, n}}$ converge to $\widehat{\mu_{1}}$ uniformly on $A_{\alpha}$. Hence,

$$
\lim _{r \rightarrow+\infty} \widehat{\mu_{1}}\left(r e^{i \theta}\right)=0 \text { for }-\pi<\theta<0 .
$$

Since inf $\operatorname{supp}\left(\mu_{j}\right)=0$ and $\inf \operatorname{supp}\left(\mu_{j}^{\prime}\right) \geqslant 0$,

each $\widehat{\mu_{j}}$ and each $\widehat{\mu_{j}^{\prime}}$ is bounded on the half-plane $\{z \in \mathbb{C}: \operatorname{Im} z \leqslant 0\}$.

For convenience of the notation, we denote $f_{j}=\widehat{\mu}_{j}$ for $1 \leqslant j \leqslant n$. Differentiating the integral defining $\widehat{\mu_{j}}$, we see that $i \widehat{\mu_{j}^{\prime}}$ is the derivative of $\widehat{\mu_{j}}$ :

$$
\widehat{\mu_{j}^{\prime}}=-i f_{j}^{\prime}
$$

Since the Fourier transform of the convolution of measures is the product of their Fourier transforms, we have

$$
\widehat{v_{j}}=-i f_{1} \ldots f_{j-1} f_{j}^{\prime} f_{j+1} \ldots f_{n} .
$$

It immediately follows that

$$
\widehat{v}=-i f_{1} \ldots f_{n} \sum_{j=1}^{n} c_{j} \frac{f_{j}^{\prime}}{f_{j}} .
$$

Since inf $\operatorname{supp}(v)>0$, there are $a, c>0$ such that

$$
\left|\widehat{v}\left(r e^{i \theta}\right)\right| \leqslant c e^{a r \sin \theta} \text { for }-\pi \leqslant \theta \leqslant 0 \text { and } r \geqslant 0 .
$$

Pick $\theta \in(-\pi, 0)$ such that the ray $\ell=\left\{r e^{i \theta}: r>0\right\}$ is free of zeros of the entire functions $f_{j}$. Then, there is a connected and simply connected open set $U \subset \mathbb{C}$ such that $\ell \subset U$ and $f_{j}$ have no zeros on $U$. Then, the multi-valued holomorphic function $f_{1}^{c_{1}} \ldots f_{n}^{c_{n}}$ splits over $U$ and we can pick its holomorphic branch $\varphi: U \rightarrow \mathbb{C}$. Differentiating and using (3.5), we obtain

$$
\varphi^{\prime}=\varphi \sum_{j=1}^{n} c_{j} \frac{f_{j}^{\prime}}{f_{j}}=\frac{i \varphi \widehat{v}}{f_{1} \ldots f_{n}} .
$$

Using the definition of $\varphi$ and the above display, we have

$$
\left|\varphi^{\prime}(z)\right|=|\widehat{v}(z)|\left|f_{1}(z)\right|^{c_{1}-1} \ldots\left|f_{n}(z)\right|^{c_{n}-1} \text { and }|\varphi(z)|=\left|f_{1}(z)\right|^{c_{1}} \ldots\left|f_{n}(z)\right|^{c_{n}},
$$


for each $z \in U$. Since $c_{j}>0$, (3.3), (3.4) and the second equality in (3.7) show that

$$
\left|\varphi\left(r e^{i \theta}\right)\right| \rightarrow 0 \text { as } r \rightarrow+\infty .
$$

Since $c_{j}>1$, (3.6), (3.4) and the first equality in (3.7) imply that there is $b>0$ such that

$$
\left|\varphi^{\prime}\left(r e^{i \theta}\right)\right| \leqslant b e^{a r \sin \theta} \text { for each } r \geqslant 0 .
$$

According to (3.8) and (3.9),

$$
\varphi\left(r e^{i \theta}\right)=-e^{-i \theta} \int_{r}^{\infty} \varphi^{\prime}\left(\rho e^{i \theta}\right) d \rho,
$$

and therefore using (3.9) once again, we get

$$
\left|\varphi\left(r e^{i \theta}\right)\right| \leqslant b \int_{r}^{\infty} e^{a \rho \sin \theta} d \rho=d e^{a r \sin \theta} \text { for all } r>0,
$$

where $d=\frac{-b}{a \sin \theta}$. Hence,

$$
\varlimsup_{r \rightarrow+\infty} \frac{\ln \left|\varphi\left(r e^{i \theta}\right)\right|}{r} \leqslant a \sin \theta<0 .
$$

On the other hand, by (3.2), there are open subsets $E_{1}, \ldots, E_{n}$ of $(0, \infty)$ of density 1 such that $\frac{\ln \left|f_{j}\left(r r^{i \theta}\right)\right|}{r} \rightarrow 0$ as $r \rightarrow+\infty, r \in E_{j}$. Since $E=E_{1} \cap \ldots \cap E_{n}$ is also a set of density $1, E$ is unbounded and $\frac{\ln \left|f_{j}\left(r^{i \theta}\right)\right|}{r} \rightarrow 0$ as $r \rightarrow+\infty, r \in E$ for $1 \leqslant j \leqslant n$. Since $\ln \left|\varphi\left(r e^{i \theta}\right)\right|=\sum_{j=1}^{n} c_{j} \ln \left|f_{j}\left(r e^{i \theta}\right)\right|$, we arrive to

$$
\lim _{\substack{r \rightarrow+\infty \\ r \in E}} \frac{\ln \left|\varphi\left(r e^{i \theta}\right)\right|}{r}=0,
$$

which contradicts (3.10). The proof is complete.

4. Proof of Theorem 1.1. Since the interior of the closure of a projective orbit of a tuple of commuting continuous linear operators does not change if we remove the operators with non-dense range from the tuple, we can, without loss of generality, assume that the operators $T_{j}$ in Theorem 1.1 have dense range. Thus, Theorem 1.1 is a corollary of the following more general result:

THEOREM 4.1. Let $\left\{T^{[t]}\right\}_{t \in \mathbb{R}_{+}^{k} \times \mathbb{Z}_{+}^{m}}$ be an operator $(k, m)$-semigroup on $L^{1}[0,1]$ consisting of truncated convolution operators with dense range. Then, for any $f \in L^{1}[0,1]$, the projective orbit

$$
O=\left\{w T^{[t]} f: w \in \mathbb{K}, t \in \mathbb{R}_{+} \times \mathbb{Z}_{+}^{m}\right\}
$$

is nowhere dense in $L^{1}[0,1]$ with respect to the weak topology.

Proof. Assume the contrary. That is, $O$ is somewhere dense. We can also assume that the number $k+m$ is minimal possible for which there exists an operator $(k, m)$ semigroup on $L^{1}[0,1]$ of truncated convolution operators with dense range possessing a somewhere dense projective orbit. Next, the infimum $c$ of the support of $f$ must equal to 
0 . Indeed, otherwise $O$ is nowhere dense as a subset of the proper closed linear subspace $L$ of $g \in L^{1}[0,1]$ vanishing on $[0, c]$. Let $M: L^{1}[0,1] \rightarrow L^{1}[0,1]$ be the multiplication by the argument operator $M h(x)=x h(x)$. Since the infimum of the support of $M f$ is also 0, Lemma 2.3 provides truncated convolution operators $B$ and $C$ with dense range such that $C M f=B f$. By Lemmas 3.2 and 3.3, we can, without loss of generality, assume that $1 \leqslant j \leqslant k$ and $T_{j}^{t}=e^{t A_{j}}$ with a quasi-nilpotent $A_{j} \in \mathbb{A}$ for each invertible $T_{j}$. In particular, each $T_{j}$ with $j>k$ is non-invertible. Denote $S_{j}=\left[T_{j}, M\right]$ for $1 \leqslant j \leqslant m+k$. By Lemma 3.1, $T_{j}=C_{\mu_{j}}$ for $1 \leqslant j \leqslant m+k$ with $\mu_{j} \in \mathbf{M}$ and $\inf \operatorname{supp}\left(\mu_{j}\right)=0$. By Lemma 3.4, $S_{j}=C_{\mu_{i}^{\prime}}$, where $\mu^{\prime}$ is the measure absolutely continuous with respect to $\mu$ with the density $\rho(x)=-x$. If the convex span of the operators $R_{1}, \ldots, R_{m+k}$ with

$$
R_{j}=T_{1} \ldots T_{j-1} S_{j} T_{j+1} \ldots T_{k+m}
$$

does not contain the zero operator, Lemma 2.5 with $\mathbb{B}=\mathbb{A}$ guarantees that $O$ is nowhere dense. This contradiction shows that 0 is in the convex span of $R_{j}$. Then, there are $1 \leqslant j_{1}<\ldots<j_{r} \leqslant k+m$ and $c_{1}, \ldots, c_{r}>0$ such that $c_{1} R_{j_{1}}+\cdots+c_{r} R_{j_{r}}=$ 0 . Since each $T_{j}$ has dense range, the last equality and the definition of $R_{j}$ imply that

$$
c_{1} R_{1}^{\prime}+\cdots+c_{r} R_{r}^{\prime}=0 \text {, where } R_{l}^{\prime}=T_{j_{1}} \ldots T_{j_{l-1}} S_{j_{l}} T_{j_{l+1}} \ldots T_{j_{r}} .
$$

Since $R_{l}^{\prime}=C_{v_{l}}$ with $v_{l}$ being the restriction to $[0,1)$ of the convolution

$$
v_{l}=\mu_{j_{1}} * \ldots * \mu_{j_{l-1}} * \mu_{j_{l}}^{\prime} * \mu_{j_{l+1}} * \ldots * \mu_{j_{r}},
$$

the equality $c_{1} R_{1}^{\prime}+\cdots+c_{r} R_{r}^{\prime}=0$ implies that the infimum of the support of $c_{1} v_{1}+$ $\cdots+c_{r} v_{r}$ is at least 1 . By Lemma 3.6, $\mu_{j_{l}}(\{0\}) \neq 0$ for $1 \leqslant l \leqslant r$. By Lemma 3.1, each $T_{j_{l}}$ is invertible. Hence, $1 \leqslant j_{l} \leqslant k$ and $T_{j_{l}}^{t}=e^{t A_{j_{l}}}$ for $1 \leqslant l \leqslant r$ and $t \in \mathbb{R}_{+}$with $A_{j_{l}} \in \mathbb{A}$ being quasi-nilpotent. Re-arranging the order of $T_{j}$ with $1 \leqslant j \leqslant k$, if necessary, we can, without loss of generality, assume that $j_{l}=l$ for $1 \leqslant l \leqslant r$. That is, $T_{j}^{t}=e^{t A_{j}}$ for $1 \leqslant j \leqslant r$ with quasi-nilpotent $A_{j} \in \mathbb{A}$. It is easy to verify that

$$
S_{j}=\left[T_{j}, M\right]=\left[e^{A_{j}}, M\right]=e^{A_{j}}\left[A_{j}, M\right]=T_{j}\left[A_{j}, M\right] \text { for } 1 \leqslant j \leqslant r .
$$

Thus, the equality $c_{1} R_{1}^{\prime}+\cdots+c_{r} R_{r}^{\prime}=0$ can be rewritten as $T_{1} \ldots T_{r}\left(c_{1}\left[A_{1}, M\right]+\right.$ $\left.\cdots+c_{r}\left[A_{r}, M\right]\right)=0$. Since $T_{j}$ are invertible for $1 \leqslant j \leqslant r$, we have $\left[c_{1} A_{1}+\cdots+\right.$ $\left.c_{r} A_{r}, M\right]=0$. By Corollary 3.5, $c_{1} A_{1}+\cdots+c_{r} A_{r}=c I$ with $c \in \mathbb{K}$. Since $A_{j}$ commute and are quasi-nilpotent, $c_{1} A_{1}+\cdots+c_{r} A_{r}$ is also quasi-nilpotent and therefore $c=0$. Thus, $c_{1} A_{1}+\cdots+c_{r} A_{r}=0$. Hence, the $\mathbb{R}$-linear span of $A_{1}, \ldots, A_{r}$ coincides with the $\mathbb{R}$-linear span of $A_{2}, \ldots, A_{r}$. Thus,

$$
\begin{aligned}
& \left\{T^{[t]}: t \in \mathbb{R}_{+}^{k} \times \mathbb{Z}_{+}^{m}\right\} \subseteq \mathcal{M}, \text { where } \\
& \mathcal{M}=\left\{e^{\tau_{1} A_{2}} \ldots e^{\tau_{r-1} A_{r}} T_{r+1}^{s_{1}} \ldots T_{k}^{s_{k-r}} T_{k+1}^{q_{1}} \ldots T_{k+m}^{q_{m}}: \tau \in \mathbb{R}^{r-1}, s \in \mathbb{R}_{+}^{k-r}, q \in \mathbb{Z}_{+}^{m}\right\} .
\end{aligned}
$$

Hence, the semigroup $\mathcal{M}$ admits a somewhere dense projective orbit. Since $\mathcal{M}$ is the union of $2^{r-1}$ subsemigroups $\mathcal{M}_{\varepsilon}$ with $\varepsilon \in\{-1,1\}^{r-1}$, where

$$
\mathcal{M}_{\varepsilon}=\left\{e^{\tau_{1} \varepsilon_{1} A_{2}} \ldots e^{\tau_{r-1} \varepsilon_{r-1} A_{r}} T_{r+1}^{s_{1}} \ldots T_{k}^{s_{k-r}} T_{k+1}^{q_{1}} \ldots T_{k+m}^{q_{m}}: \tau \in \mathbb{R}_{+}^{r-1}, s \in \mathbb{R}_{+}^{k-r}, q \in \mathbb{Z}_{+}^{m}\right\},
$$

at least one of the semigroups $\mathcal{M}_{\varepsilon}$ admits a somewhere dense projective orbit. Since each $\mathcal{M}_{\varepsilon}$ is an operator $(k-1, m)$-semigroup of truncated convolution operators with dense range, we have arrived to a contradiction with the minimality of $k+m$. 
5. Proof of Theorem 1.5. Throughout this section, we use the following notation. For $a \in L^{1}[0,1], v_{a}$ is the absolutely continuous measure on [0,1] with density $a$ and $R_{a}=I+C_{v_{a}}=C_{\delta+v_{a}}$. Of course, each $R_{a}$ is a truncated convolution operator.

LEMMA 5.1. Both sets

$$
\begin{aligned}
A & =\left\{(a, f) \in L^{1}[0,1] \times C_{0}[0,1]:\left\|R_{a}^{n} f\right\|_{1} \rightarrow \infty\right\} \\
\text { and } B & =\left\{(a, f) \in L^{1}[0,1] \times C_{0}[0,1]:\left\|R_{a}^{n} f\right\|_{\infty} \rightarrow 0\right\}
\end{aligned}
$$

are dense in the Banach space $L^{1}[0,1] \times C_{0}[0,1]$.

First, we shall prove Theorem 1.5 assuming Lemma 5.1 and we shall prove the latter afterwards.

Reduction of Theorem 1.5 to Lemma 5.1. For $n \in \mathbb{N}$, let

$$
\begin{aligned}
A_{n} & =\bigcup_{k>n}\left\{(a, f) \in L^{1}[0,1] \times C_{0}[0,1]:\left\|R_{a}^{k} f\right\|_{1}>n\right\} \\
\text { and } B_{n} & =\bigcup_{k>n}\left\{(a, f) \in L^{1}[0,1] \times C_{0}[0,1]:\left\|R_{a}^{k} f\right\|_{\infty}<n^{-1}\right\}
\end{aligned}
$$

It is easy to see that the sets $A_{n}$ and $B_{n}$ are open. Moreover, $A \subseteq A_{n}$ and $B \subseteq B_{n}$ for each $n \in \mathbb{N}$, where $A$ and $B$ are defined in Lemma 5.1. By Lemma 5.1, $A_{n}$ and $B_{n}$ are dense in $L^{1}[0,1] \times C_{0}[0,1]$ for every $n \in \mathbb{N}$. By the Baire theorem, $\Omega=\bigcap_{n=1}^{\infty}\left(A_{n} \cap B_{n}\right)$ is a dense $G_{\delta}$-subset of $L^{1}[0,1] \times C_{0}[0,1]$. In particular, $\Omega$ is non-empty and we can pick $(a, f) \in \Omega$. By the definition of $\Omega, \overline{\lim }_{n \rightarrow \infty}\left\|R_{a}^{n} f\right\|_{1}=\infty$ and $\underline{\lim }_{n \rightarrow \infty}\left\|R_{a}^{n} f\right\|_{\infty}=0$. Thus, the truncated convolution operator $T=R_{a}$ and $f \in C_{0}[0,1]$ satisfy all desired conditions.

The proof of Theorem 1.5 will be complete if we prove Lemma 5.1. The proof of the latter is based upon the following two theorems proved in [3, Theorems 1.2 and 1.3].

Theorem A. Let $r>0, W \in \mathbb{A}$ be quasi-nilpotent, $1 \leqslant p \leqslant \infty, b>0,-\pi \leqslant \alpha \leqslant$ $\pi$ and $T=I+V^{r}\left(b e^{i \alpha} I+W\right)$, where $V^{r}$ is the Riemann-Liouville operator. Then, for each non-zero $f \in L^{p}[0,1]$,

$$
\lim _{n \rightarrow \infty} \frac{\ln \left\|T^{n} f\right\|_{p}}{n^{1 /(r+1)}}=(r+1) b^{1 /(r+1)}\left(\frac{1-\inf \operatorname{supp}(f)}{r}\right)^{r /(r+1)} \cos _{+}\left(\frac{\alpha}{r+1}\right)
$$

where $\cos _{+}(t)=\max \{\cos t, 0\}$. Furthermore, the norms $\left\|T^{n}\right\|_{p}$ of the operators $T^{n}$ on the Banach space $L^{p}[0,1]$ satisfy

$$
\lim _{n \rightarrow \infty} \frac{\ln \left\|T^{n}\right\|_{p}}{n^{1 /(r+1)}}=(r+1) b^{1 /(r+1)} \cos _{+}\left(\frac{\alpha}{r+1}\right) .
$$

TheOREM B. Let $c>0,1 \leqslant p \leqslant \infty, V$ be the Volterra operator and let $X$ be the set of positive monotonically non-increasing sequences $a=\left\{a_{n}\right\}_{n=0}^{\infty}$ such that $\sum_{n=1}^{\infty} \frac{\ln a_{n}}{n^{3 / 2}}>$ $-\infty$. Then, for any non-zero $f \in L_{p}[0,1]$, there exists $a \in X$ for which $a_{n} \leqslant \|(I-$ $c V)^{n} f \|_{p}$ for each $n \in \mathbb{N}$. Conversely, for any $a \in X$, there exists a non-zero $f \in L_{p}[0,1]$ for which $\left\|(I-c V)^{n} f\right\|_{p} \leqslant a_{n}$ for each $n \in \mathbb{N}$. Moreover, if $1 \leqslant p<\infty$, then the set of $f \in L_{p}[0,1]$ for which $\left\|(I-c V)^{n} f\right\|_{p}=O\left(a_{n}\right)$ is dense in $L_{p}[0,1]$. 
Proof of Lemma 5.1. Take $a(x)=1+a_{1} x+\cdots+a_{n} x^{n}$ being a polynomial with the constant term 1 and $f$ being any non-zero function from $C_{0}[0,1]$. Then, $0 \leqslant s<$ 1 , where $s=\inf \operatorname{supp}(f) \in[0,1)$. It is easy to see that $R_{a}=I+V+\frac{a_{1}}{2} V^{2}+\cdots+$ $\frac{a_{n}}{n !} V^{n+1}$. Hence, $R_{a}=I+V(I+W)$, where $W$ is a quasi-nilpotent operator from $\mathbb{A}$. By Theorem A with $b=r=p=1$ and $\alpha=0$,

$$
\lim _{n \rightarrow \infty} \frac{\ln \left\|R_{a}^{n} f\right\|_{1}}{n^{1 / 2}}=2(b(1-s))^{1 / 2}>0 .
$$

It immediately follows that $\left\|R_{a}^{n} f\right\|_{1} \rightarrow \infty$. Thus, $(a, f) \in A$ for every non-zero $f \in$ $C_{0}[0,1]$ and every polynomial $a$ with the constant term 1 . Since the set of such polynomials is dense in $L^{1}[0,1], A$ is dense in $L^{1}[0,1] \times C_{0}[0,1]$.

Assume now that $a(x)=-1+a_{1} x+\cdots+a_{n} x^{n}$ is a polynomial with the constant term -1 . Then, $R_{a}=I-V+\frac{a_{1}}{2} V^{2}+\cdots+\frac{a_{n}}{n !} V^{n+1}=(I-V)\left(I+z V^{k}(I+W)\right)$ with $z \in \mathbb{K} \backslash\{0\}, k \geqslant 2$ and $W$ being a quasi-nilpotent operator from $\mathbb{A}$. Pick $b>0$ and $\alpha \in(-\pi, \pi]$ such that $z=b e^{i \alpha}$. Then, $R_{a}=(I-V) T$, where $T=I+b e^{i \alpha} V^{k}(I+W)$. By Theorem A,

$$
\lim _{n \rightarrow \infty} \frac{\ln \left\|T^{n}\right\|_{\infty}}{n^{1 /(k+1)}}=(k+1) b^{1 /(k+1)} \cos \left(\frac{\alpha}{k+1}\right) .
$$

Pick your favourite numbers $c$ and $d$ such that $\frac{1}{3}<c<d<\frac{1}{2}$ and consider the sequence $s_{n}=e^{-n^{d}}$ for $n \in \mathbb{N}$. Since $d<\frac{1}{2}, \sum_{n=1}^{\infty} \frac{\ln s_{n}}{n^{3 / 2}}>-\infty$ and therefore Theorem B implies that the set

$$
M=\left\{g \in L^{1}[0,1]:\left\|(I-V)^{n} g\right\|_{1}=O\left(s_{n}\right)\right\}
$$

is a dense subset of $L^{1}[0,1]$. Since $V: L^{1}[0,1] \rightarrow C_{0}[0,1]$ is a bounded linear map with dense range, $V(M)$ is a dense subset of $C_{0}[0,1]$. Since for every $g \in M, \|(I-$ $V)^{n} V g\left\|_{\infty} \leqslant\right\|(I-V)^{n} g \|_{1}$ ( $V$ has norm 1 as an operator from $L^{1}[0,1]$ to $\left.C_{0}[0,1]\right)$, we see that $\left\|(I-V)^{n} f\right\|_{\infty}=O\left(s_{n}\right)$ for every $f \in V(M)$. Hence,

$\left\|R_{a}^{n} f\right\|_{\infty}=\left\|T^{n}(I-V)^{n} f\right\|_{\infty} \leqslant\left\|T^{n}\right\|_{\infty}\left\|(I-V)^{n} f\right\|_{\infty}=O\left(s_{n}\left\|T^{n}\right\|_{\infty}\right)$ for each $f \in V(M)$.

Since $k \geqslant 2$ and $c>\frac{1}{3}$, from (5.1), it follows that $\left\|T^{n}\right\|_{\infty}=O\left(e^{n^{c}}\right)$. Since $s_{n}=e^{-n^{d}}$, by the above display, $\left\|R_{a}^{n} f\right\|_{\infty}=O\left(e^{n^{c}-n^{d}}\right)$. Since $d>c, e^{n^{c}-n^{d}} \rightarrow 0$ and therefore $\left\|R_{a}^{n} f\right\|_{\infty} \rightarrow 0$ for $f \in V(M)$. Thus, $(a, f) \in B$ if $f \in V(M)$ and $a$ is a polynomial with the constant term -1 . Since the set of such polynomials is dense in $L^{1}[0,1]$ and $V(M)$ is dense in $C_{0}[0,1], B$ is dense in $L^{1}[0,1] \times C_{0}[0,1]$.

The following questions remain open:

Question 5.2. Does there exist a truncated convolution operator $T$ on $L^{2}[0,1]$ such that every non-zero $f \in L^{2}[0,1]$ is an irregular vector for $T$ ?

As we have mentioned, for $1<p<\infty$, there are continuous linear operators on $L^{p}[0,1]$ commuting with $V$ other than truncated convolutions. Thus, the following question remains open (although probably a negative answer could be obtained by a not so sophisticated modification of the proof of Theorem 1.1).

Question 5.3. Let $1<p<\infty$. Does there exist a weakly supercyclic tuple of continuous linear operators on $L^{p}[0,1]$ commuting with $V$ ? 
ACKNOWLEDGEMENTS. The author would like to thank the referee for helpful comments.

\section{REFERENCES}

1. F. Bayart and E. Matheron, Dynamics of linear operators (Cambridge University Press, Cambridge, 2009).

2. B. Beauzamy, Introduction to operator theory and invariant subspaces (North-Holland, Amsterdam, 1988).

3. S. Bermudo, A. Montes-Rodríguez and S. Shkarin, Orbits of operators commuting with the Volterra operator, J. Math. Pures. Appl. 89 (2008), 145-173.

4. S. Eveson, Non-supercyclicity of Volterra convolution and related operators, Integral Equations Operator Theory 62 (2008), 585-589.

5. N. Feldman, Hypercyclic tuples of operators and somewhere dense orbits, J. Math. Anal. Appl. 346 (2008), 82-98.

6. E. Gallardo-Gutiérrez and A. Montes-Rodríguez, The Volterra operator is not supercyclic, Integral Equations Operator Theory 50 (2004), 211-216.

7. F. Léon-Saavedra and A. Piqueras-Lerena, Cyclic properties of Volterra Operator II [preprint].

8. B. Levin, Distribution of zeros of entire functions (AMS, Providence, Rhode Island, 1980).

9. A. Montes-Rodríguez and S. Shkarin, Non-weakly supercyclic operators, J. Operator Theory $\mathbf{5 8}$ (2007), 39-62.

10. A. Montes-Rodríguez and S. Shkarin, New results on a classical operator, Contemp. Math. 393 (2006), 139-158.

11. G. Prajitura, Irregular vectors of Hilbert space operators, J. Math. Anal. Appl. 354 (2009), 689-697.

12. H. Schäfer, Topological vector spaces (Macmillan, New York, 1966)

13. S. Shkarin, Antisupercyclic operators and orbits of the Volterra operator, J. Lond. Math. Soc. 73 (2006), 506-528.

14. S. Shakrin, Operators, commuting with the Volterra operator, are not weakly supercyclic, Integral Equations and Operator Theory 68 (2010), 229-241.

15. L. Smith, A nonhypercyclic operator with orbit-density properties, Acta Sci. Math. (Szeged) 74 (2008), 741-754. 